\title{
Determinantes de la competitividad del sector español de la informática industrial, 1995-2015. Un análisis de datos de panel
}

\author{
Miguel Ángel Sánchez Jiménez \\ miguelangel.sanchez@uca.es \\ Profesor doctor de la Universidad de Cádiz, Avenida Segunda Aguada N12. 4B, CP: 11012, \\ Cádiz (Cádiz) España
}

DOI: $10.17013 /$ risti.27.52-66

\begin{abstract}
Resumen: el objetivo de la presente investigación consiste en dilucidar los factores que determinan la posición competitiva del sector de la informática industrial de España en la eurozona en el período 1995-2015. Para alcanzar dicho objetivo, se adopta el enfoque teórico de la ventaja absoluta de coste Shaikh (2016), quien postula que los términos reales de intercambio entre las naciones se encuentran regulados en el largo plazo por los costes laborales unitarios reales de las empresas de los sectores de bienes transables. La hipótesis de la investigación es que la posición competitiva del sector de la informática industrial de España se encuentra determinada en el largo plazo por los costes laborales unitarios reales relativos. Para contrastar dicha hipótesis, se llevan a cabo pruebas de raíces unitarias y de cointegración, y se aplica la prueba de causalidad de Granger.
\end{abstract}

Palabras-clave: ventaja absoluta de coste; términos reales de intercambio; informática industrial; España; eurozona.

\section{Determinants of the competitiveness of the Spanish sector of industrial computing, 1995-2015. A panel data analysis}

\begin{abstract}
The main aim of this research is to explain the factors that govern the competitive position of the sector of industrial computing in Spain during the period 1995-2015. To achieve this objective, we adopt the Shaikh (2016) theoretical approach of the absolute cost advantage, which postulates that the real terms of exchange between nations are regulated in the long term by the real unit labor costs of the companies from tradable goods sectors. The hypothesis of the research is that the competitive position of the Spanish sector of industrial computing is determined in the long term by the relative real unit labor costs. To test this hypothesis, unit root and cointegration tests are carried out, and the Granger causality test is applied.
\end{abstract}

Keywords: Absolute cost advantage; real terms of exchange; industrial computing; Spain; Eurozone. 


\section{Introducción}

La Unión Económica y Monetaria de Europa se ha caracterizado en las dos últimas décadas por las fuertes asimetrías económicas y sociales entre los países miembros que la conforman. El proceso de convergencia de Europa hubo de interrumpirse a partir de 1993 con la libre circulación de mercancías, personas y capitales, tanto más cuanto que el déficit comercial de las economías del sur de Europa con respecto a las economías europeas noroccidentales no ha dejado de aumentar desde dicha fecha.

Sírvanse de ejemplo los siguientes datos de Eurostat (2017). En 1995, el superávit de la balanza comercial de bienes intraUE de Alemania y de Holanda alcanzaba, respectivamente, los 20.150 millones de euros corrientes y los 18.521 millones de euros corrientes. Para 2015, el superávit intraUE de estos dos países es 72.525 millones de euros corrientes y 176.342 millones de euros corrientes, respectivamente.

El superávit de ambos países se comprende en la medida en que el valor de sus exportaciones supera ampliamente el valor de sus importaciones, por cuanto son economías especializadas en la producción de bienes con alto componente tecnológico. Baste decir que, entre estos sectores, destaca el de la informática industrial cuyo desarrollo en España, aunque notable, no ha alcanzado el mismo dinamismo que en Alemania y Holanda.

El objetivo de la presente investigación consiste, pues, en dilucidar los factores que determinan la posición competitiva del sector de la informática industrial de España en el contexto de la eurozona para el período 1995-2015. Para alcanzar dicho objetivo, se adopta el enfoque teórico de la ventaja absoluta de coste Shaikh (2016), quien postula que los términos reales de intercambio entre naciones se encuentran regulados en el largo plazo por los costes laborales unitarios reales de las empresas de los sectores de bienes transables.

La novedad de este trabajo en relación con otras investigaciones que han verificado la teoría de la ventaja absoluta de coste (Guerrero, 1995; Martínez-Hernández, 2010, 2017; Góchez \& Tablas, 2013; Shaikh, 2016; Boundi, 2017, 2018a, 2018b), es que se trata del primer análisis que utiliza los datos de la base EU Klems (2017) para el sector de la informática industrial y la eurozona.

La investigación se compone de cuatro apartados. El primero corresponde al marco teórico. El segundo desarrolla las principales cuestiones metodológicas para el análisis de los resultados. El tercero está dedicado al análisis econométrico. Por último, se extraen las principales conclusiones.

\section{Marco teórico}

Para la cabal comprensión del enfoque teórico del comercio internacional con base en la ventaja absoluta de coste de Shaikh (2016), resulta pertinente desarrollar sucintamente la teoría de la competencia real. Shaikh (2016), siguiendo a Adam Smith (1776), Ricardo (1821) y Schumpeter (1942), delimita dos dimensiones de la competencia, a saber: la competencia intrasectorial y la competencia intersectorial. 
Estas dos unidades de estudio revelan que, por definición, la competencia es un proceso dinámico que tiene como característica fundamental las asimetrías productivas entre las empresas y los sectores derivadas del movimiento del capital (Boundi, 2018b). En el interior de un sector, lo que prevalece es la desigualdad de las tasas de rentabilidad empresariales merced a las disimilitudes en las estructuras de costes entre las empresas más eficientes y las rezagadas.

En cada sector existe un conjunto de empresas que actúan como las reguladoras, por cuanto poseen las condiciones técnicas de producción mejor reproducibles. $\mathrm{O}$, más precisamente, los costes unitarios de producción de dichas empresas actúan como el centro de gravitación sobre el que tenderán a orbitar en el largo plazo los precios de mercado de un sector. Esto quiere decir que las empresas menos eficientes y con estructuras de costes más caras se encuentran obligadas a vender sus bienes al precio de mercado que determinan las empresas reguladoras, aun cuando ello les comporte incurrir en pérdidas.

Esta tendencia hacia la igualación de los precios de mercado tiene como correlato que las empresas más eficientes de un sector obtendrán una mayor cuota de mercado que les garantizará márgenes de beneficio elevados respecto a sus competidores. A partir de este punto se desprende la competencia intersectorial, tanto más cuanto que el movimiento del capital implica que los flujos de las nuevas inversiones se dirigirán a los sectores con las tasas de rentabilidad más altas, abandonado así a los sectores menos rentables.

Del excepcional aumento del volumen de inversión en los sectores dinámicos sobrevendrá el crecimiento de la producción por encima de la demanda, provocando el descenso de los precios de mercado hasta situarse en el nivel del precio de producción, en cuanto precio que garantiza la obtención de la tasa de rentabilidad promedio. En los sectores menos eficientes ocurre lo contrario. La fuga del capital deprime la oferta por debajo de la demanda, elevando el precio de mercado hasta converger hacia el precio de producción.

Grosso modo, en tanto que la competencia intrasectorial tiende a igualar los precios de mercado, la competencia intersectorial nivela tendencialmente las tasas de rentabilidad. Vale decir que estas dos tendencias en apariencia contradictorias coexisten en la medida en que el movimiento intersectorial del capital se encuentra regulado por las tasas de rentabilidad de las empresas mejor dotadas técnicamente de cada sector.

En tal sentido, dentro de las fronteras de un país los precios de mercado relativos de dos bienes cualesquiera se encuentran regidos por los precios de producción de las empresas reguladoras de cada sector:

$$
\frac{P_{i}}{P_{j}} \cong \frac{P_{i}^{*}}{P_{i}^{*}}
$$

Donde:

$P_{i}=$ precio de mercado del $i$-enésimo bien.

$P_{j}=$ precio de mercado del $j$-enésimo bien. 
$P_{i}^{*}=$ precio de producción de la empresa reguladora del $i$-enésimo sector.

$P_{i}^{*}=$ precio de producción de la empresa reguladora del $j$-enésimo sector.

Tal y como señala Shaikh (2016), el precio de producción de cada empresa reguladora depende de sus costes laborales unitarios reales (en adelante, CLUR):

Donde:

$$
\frac{P_{i}^{*}}{P_{i}^{*}} \cong \frac{C L U R_{i}^{*}}{C L U R_{j}^{*}} \cong \frac{\frac{W_{r i}^{*}}{Q_{r i}^{*}}}{\frac{W_{r j}^{*}}{Q_{r j}^{*}}}
$$

$C L U R_{i}^{*}=$ coste laboral unitario real de la empresa reguladora del $i$-enésimo sector.

$C L U R_{j}^{*}=$ coste laboral unitario real de la empresa reguladora del $j$-enésimo sector.

$W_{r i}^{*}=$ salario real de la empresa reguladora del $i$-enésimo sector.

$Q_{r i}^{*}=$ productividad real de la empresa reguladora del $i$-enésimo sector.

$W_{r j}^{*}=$ salario real de la empresa reguladora del $j$-enésimo sector.

$Q_{r j}^{*}=$ productividad real de la empresa reguladora del $j$-enésimo sector.

En consecuencia, los CLUR de las empresas más eficientes de cada sector se erigen como los reguladores de la dinámica fundamental de los precios de mercado:

$$
\frac{P_{i}}{P_{j}} \cong \frac{P_{i}^{*}}{P_{i}^{*}} \cong \frac{C L U R_{i}^{*}}{C L U R_{j}^{*}}
$$

Shaikh (2016) aduce que lo que es válido para explicar el movimiento en el largo plazo de los precios de mercado dentro de un país, también sirve para comprender su comportamiento a nivel internacional. Bajo el supuesto de que cada nación posee una empresa reguladora en aquellos sectores en los cuales son más competitivas, al incluir en la ecuación (3) el tipo de cambio nominal efectivo se tiene que las relaciones reales de intercambio estarán sujetas en el largo plazo por los CLUR relativos (en adelante, CLURR):

$$
T C R=e^{*} \frac{P_{j}^{B}}{P_{i}^{A}} \cong C L U R R=\frac{C L U R_{j}^{B}}{C L U R_{i}^{A}}
$$

Donde:

$T C R$ = tipo de cambio real efectivo del país A (nacional) respecto del país B (extranjero). $e=$ tipo de cambio nominal efectivo del país A respecto del país $\mathrm{B}$.

$P_{j}^{B}=$ precio de mercado del j-enésimo bien producido por el país B. 
$P_{i}^{A}=$ precio de mercado del i-enésimo bien producido por el país A.

$C L U R R=$ costes laborales unitarios reales relativos.

$C L U R_{j}^{B}=$ coste laboral unitario real de la empresa reguladora del j-enésimo sector del país B.

$C L U R_{i}^{A}=$ coste laboral unitario real de la empresa reguladora del i-enésimo sector del país A.

De la ecuación (4) puede inferirse que el país A mejorará su posición competitiva para con el país B, si los CLUR de la empresa reguladora del $i$-enésimo sector de A descienden con respecto a los CLUR de empresa reguladora del $j$-enésimo sector de $\mathrm{B}$, y viceversa. Luego, los términos reales de intercambio entre las naciones estarán determinados por los salarios reales y las condiciones técnicas de producción generales de cada país (Guerrero, 1995, Martínez-Hernández, 2010, 2017; Góchez \& Tablas, 2013; Shaikh, 2016; Boundi, 2017, 2018a).

A este respecto, Guerrero (1995) recalca enérgicamente que la ventaja absoluta no debe reducirse bajo ningún concepto a una ventaja absoluta de productividad, puesto que el coste de producción de una empresa se encuentra afectado conjuntamente por los salarios reales y la productividad. El país más productivo sólo será más competitivo que el país con los salarios reales más bajos, en tanto y en cuanto la diferencia en productividad sea más elevada que la diferencia en los salarios reales (Guerrero, 1995; Góchez \& Tablas, 2013; Boundi, 2017, 2018a, 2018b). De lo contario, el coste de producción será más elevado y los márgenes de beneficio más bajos en el país más productivo.

No en vano, como destacan Guerrero (1995) y Shaikh (2016), la contención de los costes de producción no sólo estriba de la habilidad de una empresa para someter el crecimiento de los salarios a un nivel inferior al de la productividad, sino también está influida por las condiciones técnicas de producción generales de un país.

Más precisamente, la productividad laboral depende tanto de la productividad del trabajo directo como de la productividad del trabajo indirecto para producir todos los bienes de capital y los bienes intermedios que requiere una empresa para elaborar sus mercancías. Id est, los aumentos de la productividad de las empresas serán mayores en una economía cuya estructura productiva esté conformada por un gran número de sectores dinámicos. Esto coadyuva a comprender las razones del porqué los países más competitivos no lo son únicamente en un sector, destacando su dominio en el mercado internacional de bienes de alto valor añadido y de alto componente tecnológico. Tales son los elementos que definen la teoría de la ventaja absoluta de Shaikh (2016), pasando a continuación a desarrollar las cuestiones metodológicas.

\section{Metodología. Hipótesis, base de datos y modelo}

Tomando como referencia el enfoque teórico de la ventaja absoluta de Shaikh (2016), se tiene a bien considerar como la hipótesis de la investigación la siguiente: la posición competitiva del sector de la informática industrial de España en el contexto de la 
eurozona se encuentra determinada en el largo plazo por los CLURR. Debe indicarse que el período de análisis corresponde al que va desde 1995 hasta 2015, puesto que la base de datos EU Klems 2017 sólo contiene la información de las variables económicas relacionadas con el sector de la informática industrial para dicho espacio temporal.

Asimismo, la hipótesis de investigación implica que se ha de verificar empíricamente que TCR del sector de la informática industrial de España respecto del sector de la informática industrial del $i$-enésimo país de la eurozona tiene como centro de gravitación los CLURR:

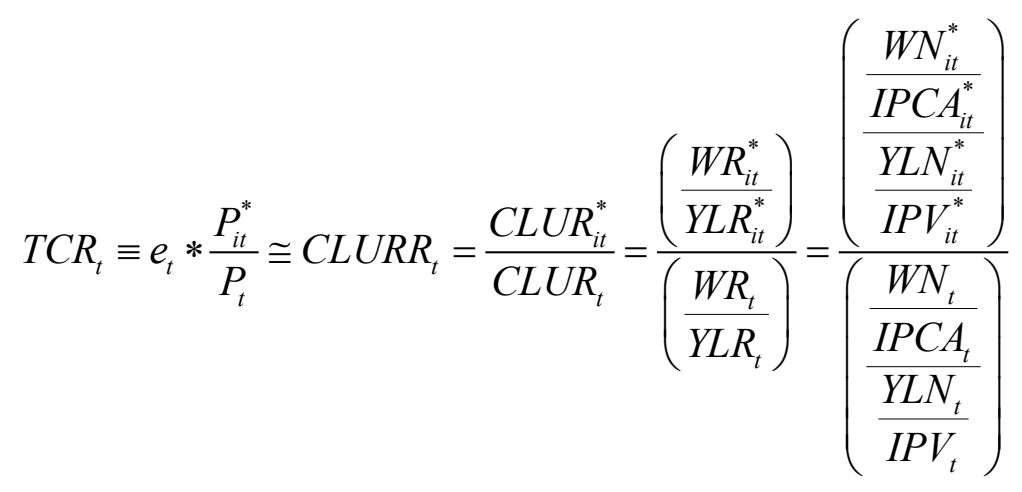

Donde:

$T C R_{t}=$ tipo de cambio real efectivo del sector de la informática industrial de España respecto del sector de la informática industrial del i-enésimo país de la eurozona en el momento $t$.

$e_{t}=$ tipo de cambio nominal efectivo del sector de la informática industrial de España respecto del sector de la informática industrial del i-enésimo país de la eurozona en el momento t.

$P_{i t}^{*}=$ precios del sector de la informática industrial del $i$-enésimo país de la eurozona en el momento t.

$P_{t}=$ precios del sector de la informática industrial de España en el momento t.

$C L U R R_{t}=$ coste laboral unitario real relativo en el momento $t$.

$C L U R_{i t}^{*}=$ coste laboral unitario real del sector de la informática industrial del $i$-enésimo país de ${ }^{i t}$ la eurozona en el momento $t$.

$C L U R_{t}=$ coste laboral unitario real del sector de la informática industrial de España en el momento $t$.

$W R_{i t}^{*}=$ salario por hora en euros constantes de 1999 del sector de la informática industrial del ${ }^{i t} i$-enésimo país de la eurozona en el momento t.

$Y L R_{i t}^{*}=$ productividad del trabajo por hora en euros constantes de 1999 del sector de la informática industrial del $i$-enésimo país de la eurozona en el momento t. 
$W R_{t}=$ salario por hora en euros constantes de 1999 del sector de la informática industrial de España en el momento t.

$Y L R_{t}=$ productividad del trabajo por hora en euros constantes de 1999 del sector de la informática industrial de España en el momento t.

$W N_{i t}^{*}=$ salario nominal por hora del sector de la informática industrial del $i$-enésimo país de la eurozona en el momento $t$.

$I P C A_{i t}^{*}=$ índice de precios al consumo armonizado del i-enésimo país de la eurozona (año base 1999).

$Y L N_{i t}^{*}=$ productividad laboral nominal por hora del sector de la informática industrial del $i$-ênésimo país de la eurozona en el momento t.

$I P V_{\text {it }}^{*}=$ índice de precios del valor añadido bruto del sector de la informática industrial del $i$-enésimo país de la eurozona en el momento t (año base 1999).

$W N_{t}=$ salario nominal por hora del sector de la informática industrial de España en el momento t.

$I P C A_{t}=$ índice de precios al consumo armonizado de España (año base 1999).

$Y L N_{t}=$ productividad laboral nominal por hora del sector de la informática industrial de España en el momento t.

$I P V_{t}=$ índice de precios del valor añadido bruto del sector de la informática industrial de España en el momento t (año base 1999).

$\mathrm{t}=(1995 \ldots 2015)$.

Llegado a este punto, debe indicarse que la determinación de las relaciones reales de intercambio entre país no es en forma alguna monocausal. De los múltiples factores que afectan a la competitividad intrasectorial y, por extensión, al TCR, se ha considerado incluir el volumen de la formación bruta de capital fijo del sector de la informática industrial de España (en adelante, FBKF). Esta variable incluye tanto la inversión en reposición como la inversión para la ampliación de maquinaria, equipos y plantas del sector.

La importancia de esta variable reside en que es un buen proxy del ciclo económico del sector, permite captar la influencia del cambio tecnológico en la competitividad intra e intersectorial, y consiente medir la elasticidad precio-cantidad los bienes de equipo. En relación con la base datos, se eligió EU Klems 2017 por las siguientes razones (O’Mahony \& Timmer, 2009; Ark \& Jäger, 2017):

i. Es compatible con el nuevo Sistema Europeo de Contabilidad Nacional (ESA 2010).

ii. Respeta la clasificación de la Nomenclatura Estadística de Actividades Económicas de la Comunidad Europea revisión 2 (NACE 2), consistente con la International Standard Industrial Classification of All Economic Activities revisión 4 (ISEC Rev. 4)

iii. Toda la información sobre las variables económicas es compatible con Eurostat. 
iv. Los datos sobre empleo están basados en la metodología de European Labour Force Survey (LFS) y de Structure of Earning Survey (SES).

v. De acuerdo con la clasificación NACE 2 que sigue EU Klems 2017, el sector de la informática industrial corresponde a (26-27) de equipos electrónicas y ópticos, (28) maquinaria y equipos informáticos, y (62-63) IT y otros servicios informáticos. Se procedió a agregar a ambos en un único sector que representa el sector de la informática industrial.

El índice de precios al consumo armonizado (IPCA) de España y de cada i-enésimo país de la eurozona se recabó de Eurostat. Vale decir que el IPCA mide el gasto del consumo de los hogares para el conjunto de la Unión Europea, tanto de los residentes como de los no residentes. De esta forma, se obtuvo la siguiente ecuación del TCR del sector de la informática industrial:

$$
T C R_{t}=f\left(C L U R R_{t}, F B K F_{t}\right)
$$

Traducida la ecuación (6) en términos econométricos, se tiene una regresión múltiple estimada por mínimos cuadrados ordinarios (MCO), donde cada una de las variables es transformada en logaritmo natural a fin de obtener la elasticidad entre ellas:

$$
L N\left(T C R_{t}\right)=\beta_{0}+\beta_{1} L N\left(C L U R R_{t}\right)+\beta_{2} L N\left(I N V_{t}\right)+\varepsilon_{t}
$$

Donde:

$L N=$ logaritmo natural o logaritmo neperiano.

$\beta_{0}=$ término constante.

$\beta_{i}=$ coeficientes múltiples de determinación.

$\varepsilon_{t}=$ término error.

Conviene remarcar que los datos para estimar la ecuación (7) se agruparon en un dato de panel, con el objetivo de verificar la existencia de una relación de cointegración entre las variables. Por consiguiente, se aplican las pruebas de raíces unitarias de Breitung (2000), de Maddala y Wu (1999) (MW) y de Hadri (2000) para comprobar que las series son no estacionarias en nivel. En la tabla 1 pueden consultarse las hipótesis nulas para cada test.

\begin{tabular}{ll}
\hline Prueba & Hipótesis nula \\
\hline Breitung (2000) & Proceso común de raíz unitaria \\
\hline Maddala y Wu (1999) & Proceso individual de raíz unitaria \\
\hline Hadri (2000) & Estacionariedad \\
\hline
\end{tabular}

Fuente: elaboración del autor

Tabla 1 - Hipótesis nulas de las pruebas de raíces unitarias 
Si los antedichos test verifican que las tres variables contienen raíz unitaria de grado I(1), se comprobará la hipótesis de cointegración por medio de las pruebas de Pedroni (1999) y de Kao (1999) basadas en el procedimiento de Engle y Granger (1987) para las series temporales. Ulteriormente, los vectores cointegración se obtendrán del MCO dinámico (DOLS) y del MCO totalmente modificado (FMOLS), tal y como recomienda la literatura sobre los datos de panel dinámicos (Stock \& Watson, 1993; Pedroni, 1999, 2002; Greene, 2003). Puesto que la correlación entre las variables no implica la existencia de relaciones de causalidad, se utiliza la prueba de Granger (1969) con base en las directrices de Wiener (1956).

\section{Análisis econométrico}

Debe indicarse que las pruebas de raíces unitarias de Breitung y de MW se realizan con un retardo siguiendo el criterio de Akaike. Por otra parte, la ecuación de las pruebas de Breitung, de MW y de Hadri contiene un intercepto individual y una tendencia. Se advierte de los resultados compendiados en la tabla 2 que en nivel las tres pruebas son no estacionarias. Esto es, las variables en nivel poseen raíz unitaria y el orden de integración es I(1), verificando de esta suerte la primera condición para la cointegración.

\begin{tabular}{|c|c|c|c|c|c|c|c|}
\hline $\begin{array}{l}\text { Variable/ } \\
\text { Prueba }\end{array}$ & $\begin{array}{l}\text { Breitung } \\
\text { (2000) }\end{array}$ & & $\begin{array}{l}\text { MW } \\
\text { (1999) }\end{array}$ & & $\begin{array}{l}\text { Hadri } \\
(2000)\end{array}$ & & \\
\hline En nivel & t-Estadístico & Prob. & $\begin{array}{l}\text { ADF- } \\
\text { Fisher X }\end{array}$ & Prob. & z-Estadístico & Prob. & Orden \\
\hline LN(TCR) & $-1,557$ & $0,060^{*}$ & 22,620 & 0,423 & 4,317 & $0,000^{* * *}$ & $\mathrm{I}(1)$ \\
\hline LN(CLURR) & 0,115 & 0,546 & 30,858 & 0,101 & 9,484 & $0,000^{* * *}$ & $\mathrm{I}(1)$ \\
\hline $\mathrm{LN}(\mathrm{FBKF})$ & $-1,100$ & 0,156 & 38,239 & 0,108 & 6,188 & $0,000^{* * *}$ & $\mathrm{I}(1)$ \\
\hline $\begin{array}{l}\text { En primera } \\
\text { diferencia }\end{array}$ & t-Estadístico & Prob. & $\begin{array}{l}\text { ADF- } \\
\text { Fisher X }\end{array}$ & Prob. & z-Estadístico & Prob. & Orden \\
\hline DLN(TCR) & $-8,135$ & $0,000^{* * *}$ & 87,783 & $0,000^{* * *}$ & 22,034 & $0,000^{* * *}$ & $\mathrm{I}(\mathrm{O})$ \\
\hline DLN(CLURR) & $-6,223$ & $0,000^{* * *}$ & 106,207 & $0,000^{* * * *}$ & 11,297 & $0,000^{* * *}$ & $\mathrm{I}(\mathrm{o})$ \\
\hline DLN(FBKF) & $-8,060$ & $0,000^{* * *}$ & 106,462 & $0,000^{* * *}$ & 2,725 & $0,032^{* * *}$ & $\mathrm{I}(\mathrm{o})$ \\
\hline
\end{tabular}

*** Denota rechazo al 1\%, al 5\% y al 10\%.

** Denota rechazo al 5\% y al 10\%.

* Denota rechazo al 10\%.

Fuente: elaboración del autor con base en Eviews9

Tabla 2 - Resultados de las pruebas de raíces unitarias

$\mathrm{Al}$ transformar las variables en sus primeras diferencias, las pruebas de Breitung y de MW indican que estas son estacionarias e integradas de orden I(o), si bien la prueba de Hadri admite el rechazo de la hipótesis de estacionariedad. En suma, existe suficiente evidencia estadística que apunta a que las variables en nivel son no estacionarias e integradas del mismo orden. 
A continuación, se procede a la interpretación de los resultados de las pruebas de cointegración de Pedroni y de Kao. Antes bien, baste recalcar que se eligió el criterio de información de Akaike para la especificación de número máximo de rezagos. Igualmente, vale la pena indicar que ambas pruebas se hicieron con un intercepto individual.

Obsérvese de la tabla 3 que la probabilidad asociada al panel rho-Estadístico, al panel PP-Estadístico, al panel ADF-Estadístico y al grupo ADF-Estadístico consiente el rechazo de la hipótesis nula de no cointegración para al menos el 10\% de nivel de significación. Empero, el panel v-Estadístico y los grupos rho-Estadístico y grupo PP-Estadístico no permiten rechazar dicha hipótesis (véase tabla 3).

Por su parte, la prueba Kao apunta al rechazo de la hipótesis nula de no cointegración para cualquier nivel de significación, en tanto y en cuanto la probabilidad vinculada al estadístico t es aproximadamente igual a cero. Por lo tanto, las pruebas de Pedroni y de Kao arrojan suficiente evidencia estadística que revela que TCR, CLURR y FBKF están cointegradas en el largo plazo.

\begin{tabular}{lllll}
\hline Pedroni (1999): Hipótesis nula = no cointegración & & & \\
\hline Dentro de las dimensiones & Estadístico & Prob. & $\begin{array}{l}\text { Tamaño del } \\
\text { estadístico }\end{array}$ & Prob. \\
\hline Panel v-Estadístico & $-1,988$ & 0,977 & $-1,020$ & 0,846 \\
\hline Panel rho-Estadístico & $-1,738$ & $0,041^{* *}$ & $-0,234$ & 0,408 \\
\hline Panel PP-Estadístico & $-2,505$ & $0,006^{* * *}$ & $-1,479$ & $0,070^{*}$ \\
\hline Panel ADF-Estadístico & $-2,470$ & $0,007^{* * *}$ & $-2,116$ & $0,017^{* *}$ \\
\hline Entre las dimensiones & Estadístico & Prob. & & \\
\hline Grupo rho-Estadístico & 1,506 & 0,934 & & \\
\hline Grupo PP-Estadístico & $-0,721$ & 0,235 & & \\
\hline Grupo ADF-Estadístico & $-1,415$ & $0,078^{*}$ & & \\
\hline
\end{tabular}

Kao (1999): Hipótesis nula = no cointegración

\begin{tabular}{lllll}
\hline & t-Estadístico & Prob. & $\begin{array}{l}\text { Varianza } \\
\text { residual }\end{array}$ & $\begin{array}{l}\text { Varianza } \\
\text { HAC }\end{array}$ \\
\hline $\mathrm{ADF}$ & $-4,790$ & $0,000^{* * *}$ & 0,615 & 0,401 \\
\hline
\end{tabular}

*** Denota rechazo al 1\%, al 5\% y al 10\%.

** Denota rechazo al 5\% y al 10\%.

* Denota rechazo al 10\%.

Fuente: elaboración del autor con base en Eviews9

Tabla 3 - Resultados de las pruebas de cointegración

Los vectores de cointegración dados por el panel DOLS y el panel FMOLS se recogen en la tabla 4. Como en los casos anteriores, el máximo número de rezagos fue seleccionado de acuerdo con el criterio de información de Akaike. En la tabla 4 se puede comprobar que la correlación entre el LN (TCR) y el LN(CLURR) es positiva, además de estadísticamente significativa al 1\%, al 5\% y al 10\%. 
Los resultados obtenidos revelan que el aumento del 1\% de LN(CLURR) provoca, ceteris paribus, la elevación de 0,657\% y de 0,680\% de LN(TCR) según el panel DOLS y el panel FMOLS, respectivamente. Concretamente, el TCR del sector de la informática industrial de España en relación con el sector de la informática industrial de cada i-enésimo país de la eurozona se depreciará por las siguientes razones.

1) Los CLUR del sector de la informática industrial de cada i-enésimo país de la eurozona se elevan y los CLUR del sector de la informática industrial se mantienen constantes. 2) Los CLUR en el sector de la informática industrial de cada i-enésimo país de la eurozona crecen en mayor proporción que el aumento de los CLUR del sector de la informática industrial de España. 3) Los CLUR en el sector de la informática industrial de cada i-enésimo país de la eurozona disminuyen en menor proporción que el descenso de los CLUR del sector de la informática industrial de España. Estos tres posibles escenarios pueden originarse en tanto que se vean afectados los salarios reales y las condiciones técnicas de producción generales en cada país de la eurozona.

Así, por ejemplo, el sector de la informática industrial de España puede beneficiarse de una política del gobierno que priorice la eficiencia energética por medio de la transición desde las energías fósiles hacia el uso más intensivo de las energías renovables. En este sentido, el abaratamiento de los costes fijos asociados al consumo de la energía devendrá en el descenso de los costes de producción de las empresas del sector de la informática industrial de España.

De igual forma, el aumento en el gasto de investigación, desarrollo e innovación $(\mathrm{I}+\mathrm{D}+\mathrm{i})$ podrá mejorar las condiciones técnicas de producción generales de la economía española, trasladándose en el aumento de la productividad laboral del sector de la informática industrial.

En relación con la contención salarial a través de las reformas laborales que buscan flexibilizar el mercado de trabajo español, estas tienen como efecto el descenso de los CLUR. Empero, el sector de la informática industrial se caracteriza por ser demandante de fuerza de trabajo altamente cualificada y, en consecuencia, es remunerada con elevados salarios (Barraza, \& Zepeda, 2017; Flores, \& Hadfeg, 2017)

Por lo anterior, debe indicarse que las empresas del sector compiten por contratar al personal mejor cualificado y más talentoso, lo cual significa que la tendencia es hacia el incremento de los salarios reales. El mantenimiento de los salarios reales a un nivel inferior que en el resto de los países la eurozona puede devenir en el brain drain, la escasez de fuerza de trabajo y el descenso de la productividad laboral para el sector en España.

Pasando a analizar la relación entre LN(TCR) y LN(FBKF), el signo positivo revela que el aumento del volumen de la inversión del sector provocará, ceteris paribus, una depreciación real del o,010\% según el panel DOLS y el panel FMOLS. Luego, se infiere que la posición competitiva del sector de la informática industrial se encuentra sujeto al ciclo económico y al gasto de inversión en bienes de capital.

Por último, se analizan los resultados de la prueba de Granger de causalidad. En la tabla 5 se observa que los t-estadísticos permiten rechazar la hipótesis nula de que LN(CLURR) y LN(FBKF) no causan, en sentido de Granger, cambios en LN(TCR). 


\begin{tabular}{|c|c|c|c|c|}
\hline \multicolumn{5}{|c|}{ Variable dependiente: LOG(TCR) } \\
\hline \multicolumn{5}{|c|}{ DOLS } \\
\hline Variable & Coeficiente & Error estándar & t-Estadístico & Prob. \\
\hline LN(CLURR) & 0,657 & 0,058 & 11,406 & $0,000^{* * *}$ \\
\hline $\mathrm{LN}(\mathrm{FBKF})$ & 0,010 & 0,004 & 2,077 & $0,040^{* *}$ \\
\hline \multicolumn{5}{|l|}{ FMOLS } \\
\hline Variable & Coeficiente & Error estándar & t-Estadístico & Prob. \\
\hline LN(CLURR) & 0,680 & 0,051 & 13,250 & $0,000^{* * *}$ \\
\hline $\mathrm{LN}(\mathrm{FBKF})$ & 0,010 & 0,032 & 2,176 & $0,030^{* *}$ \\
\hline
\end{tabular}

*** Denota rechazo al 1\%, al 5\% y al 10\%.

** Denota rechazo al 5\% y al 10\%.

* Denota rechazo al 10\%.

Fuente: elaboración del autor con base en Eviews

Tabla 4 - Resultados del panel DOLS y el panel FMOLS

Mas, por otra parte, no es factible rechazar la hipótesis nula de que LN(TCR) no causa, en sentido de Granger, variaciones en LN(CLURR) y LN(FBKF). Más aún, la prueba de Granger indica que tanto en el corto plazo (un retardo) como en el largo plazo (de dos retardos hasta cinco retardos) LN(CLURR) y LN(FBKF)provocan cambios en LN(TCR), pero no viceversa.

El análisis econométrico apunta que entre el TCR, los CLURR y la FBKF existe una relación estable en el largo plazo. Asimismo, los cambios en la posición competitiva del sector de la informática industrial se encuentran causados por los costes unitarios de producción y por el gasto de inversión en bienes de capital.

\begin{tabular}{|c|c|c|c|c|c|c|}
\hline Hipótesis nula & $\mathbf{1}$ & 2 & 3 & 4 & 5 & Relación \\
\hline $\begin{array}{l}\text { LN(CLURR) no causa en sentido de } \\
\text { Granger LN(TCR) }\end{array}$ & $15,316^{* * *}$ & $6,581^{* *}$ & $3,4115^{*}$ & $4,2442^{*}$ & $3,353^{*}$ & Causa \\
\hline $\begin{array}{l}\text { LN(TCR) no causa en sentido de } \\
\text { Granger LN(CLURR) }\end{array}$ & 0,474 & 0,62 & 0,611 & 1,436 & 1,283 & No causa \\
\hline $\begin{array}{l}\text { LN(FBKF) no causa en sentido de } \\
\text { Granger LN(TCR) }\end{array}$ & $7,446^{* * *}$ & $5,323^{*}$ & $3,0329 *$ & $2,2493^{*}$ & 1,548 & Causa \\
\hline $\begin{array}{l}\text { LN(TCR) no causa en sentido de } \\
\text { Granger LN(FBKF) }\end{array}$ & 0,87 & 0,521 & 0,781 & 2,249 & 1,297 & No causa \\
\hline
\end{tabular}

*** Denota rechazo al 1\%, al 5\% y al 10\%.

** Denota rechazo al 5\% y al 10\%.

* Denota rechazo al 10\%.

Fuente: elaboración del autor con base en Eviews9

Tabla 5 - Prueba causalidad de Granger (con 1, 2, 3, 4 y 5 retados) 


\section{Conclusiones}

En esta investigación se ha aplicado un modelo de datos de panel dinámico que ha permitido hallar la correlación y la causalidad entre el TCR, los CLURR y la FBKF del sector de la informática industrial, a lo largo del lapso que va desde 1996 hasta 2015. Toda la información fue recabada de la base de EU Klems 2017, cuya clasificación industrial se encuentra basada en NACE 2, haciendo de esta forma consistente el análisis entre España y sus socios de la eurozona.

Ello ha revelado que la peor posición competitiva de las empresas españolas cuya actividad económica se desarrolla en el sector de la informática industrial, hunde sus raíces en causas estructurales. Más exactamente, los mayores CLUR del sector español de la informática industrial nace de las peores condiciones técnicas de producción generales de España respecto de las economías de la eurozona más dinámicas.

Los recortes en $\mathrm{I}+\mathrm{D}+\mathrm{i}$, la ausencia de un programa de transición energética, la elevada carga impositiva que soportan las pequeñas y las medianas empresas de nueva creación, el deterioro de las condiciones laborales tras las reformas de 2010 y 2012 que ha provocado la migración de técnicos e ingenieros hacia otros países de la eurozona, así como la escasa inversión en infraestructura económica, actúan como limitantes para que las empresas del sector de la informática industrial mejoren su posición competitiva en la eurozona.

Aún más, la estrategia competitiva basada en la contención salarial si bien ha mejorado la capacidad de exportación de las empresas españolas en términos generales, ha supuesto la contracción del poder de compra de las familias estrechando con ello la capacidad del mercado nacional para absorber los bienes y los servicios producidos por el sector de la informática industrial.

Por lo anterior, a nivel institucional las administraciones públicas han de poner en marcha una serie de programas estructurales que consientan en el largo plazo mejorar las condiciones técnicas de producción generales de la economía española. A nivel microeconómico y mesoeconómico, las empresas del sector habrán de reducir sus CLUR mediante el desarrollo de métodos de organización productiva que haga un uso más eficiente e intensivo del capital instalado.

\section{Referencias}

Barraza, Í. D., \& Zepeda, V. V. (2017). Factores sociales y humanos que afectan el proceso de educción de requerimientos: una revisión sistemática. RISTI-Revista Ibérica de Sistemas e Tecnologias de Informação, (24), 69-83. doi: http://dx.doi. org/10.17013/risti.n.69-83

Boundi Chraki, F. (2017). Determinantes de las relaciones reales de intercambio de España con Alemania (1970-2010). Un análisis econométrico de la ventaja absoluta de costo intrasectorial. Cuadernos de economía, 36(71), 489-520. doi: https://doi. org/10.15446/cuad.econ.v36n71.48664 
Boundi Chraki, F. (2018a). Competitividad de los sectores manufactureros de Alemania y España, 1995-2015: análisis de la ventaja absoluta de coste con datos de panel dinámico. Revista Galega de Economía, 27(1), 5-16.

Boundi Chraki, F. (2018b). Desarrollo desigual y trabajo potenciado en la eurozona. Revista Problemas del Desarrollo, 49(194), 143-167. doi: http://dx.doi. org/10.22201/iiec.20078951e.2018.194.62222

Breitung, J. (2001). The local power of some unit root tests for panel data. In: T. B. Fomby, R. Carter Hill, I. Jeliazkov, J. Carlos Escanciano y E. Hillebrand (eds.) Nonstationary panels, panel cointegration, and dynamic panels (pp. 161-177). Nueva York: Emerald Group Publishing Limited.

Engle, R. F., \& Granger, C. W. J. (1987). Co-Integration and Error Correction: Representation, Estimation, and Testing. Econometrica, 55(2), 251-276.

Eurostat (2017). Globalisation patterns in EU trade and investment. Luxemburgo: European Union.

Flores, V., \& Hadfeg, Y. (2017). Un método para generar explicaciones de resultados de un Sistema Experto, usando Patrones de discurso y Ontología. RISTI-Revista Ibérica de Sistemas e Tecnologias de Informação, (21), 99-114. doi: http://dx.doi. org/0.17013/risti.21.99-114

Góchez Sevilla, R., \& Tablas, V. A. (2013). Tipo de cambio real y déficit comercial en Guatemala (1970-2007): un enfoque heterodoxo. México: CEPAL.

Granger, C. W. J. (1969). Investigating causal relations by econometric models and cross-spectral methods. Econometrica, 37(3), 424-438. doi: https://doi. org/10.2307/1912791

Guerrero, D. (1995). Competitividad: teoría y política. Barcelona: Ariel.

Hadri, K. (2000). Testing for stationarity in heterogeneous panel data. The Econometrics Journal, 3(2), 148-161. doi: https://doi.org/10.1111/1368-423X.00043

Johansen, S. (1988). Statistical analysis of cointegration vectors. Journal of economic dynamics and control, 12(2-3), 231-254. doi: https://doi.org/10.1016/01651889(88)90041-3

Johansen, S. (1991). Estimation and hypothesis testing of cointegration vectors in Gaussian vector autoregressive models. Econometrica, 59(6), 1551-1580. doi: https://doi.org/10.2307/2938278

Kao, C. (1999). Spurious regression and residual-based tests for cointegration in panel data. Journal of econometrics, 90(1), 1-44. doi: https://doi.org/10.1016/So3044076(98)00023-2

Maddala, G. S., \& Wu, S. (1999). A comparative study of unit root tests with panel data and a new simple test. Oxford Bulletin of Economics and statistics, 61(S1), 631-652. doi: https://doi.org/10.1111/1468-0084.0610s1631 
Martínez-Hernández, F. A. (2010). An alternative theory of real exchange rate determination: theory and empirical evidence for the Mexican economy, 19702004. Investigación Económica, 69(273), 55-84.

Martínez-Hernández, F. A. (2017). The Political Economy of Real Exchange Rate Behavior: Theory and Empirical Evidence for Developed and Developing Countries, 1960-2010. Review of Political Economy, 29(4), 566-596. doi: https://doi.org/10 $.1080 / 09538259.2017 .1382060$

O’Mahony, M., \& Timmer, M. P. (2009). Output, input and productivity measures at the industry level: the EU KLEMS database. The economic journal, 119(538). doi: https://doi.org/10.1111/j.1468-0297.2009.02280.x

Pedroni, P. (1999). Critical values for cointegration tests in heterogeneous panels with multiple regressors. Oxford Bulletin of Economics and statistics, 61(S1), 653-670. doi: https://doi.org/10.1111/1468-0084-0610s1653

Pedroni, P. (2001). Fully modified OLS for heterogeneous cointegrated panels. En: T. B. Fomby, R. Carter Hill, I. Jeliazkov, J. Carlos Escanciano y E. Hillebrand (eds.) Nonstationary panels, panel cointegration, and dynamic panels (pp. 93-130). Nueva York: Emerald Group Publishing Limited.

Ricardo, D. (1821). Principios de economía política y de tributación. México: FCE.

Schumpeter, J. A. (1942). Capitalism, Socialism and Democracy. Estados Unidos: Routledge.

Shaikh, A. (2016). Capitalism: Competition, conflict, crises. Nueva York: Oxford University Press.

Smith, A. (1776). La riqueza de las naciones. México: FCE.

Stock, J., \& Watson, M. (1993). A Simple Estimator of Cointegrating Vectors in Higher Order Integrated Systems. Econometrica, 61(4), 783-820. doi: https://doi. org/10.2307/2951763

van Ark, B., \& Jäger, K. (2017). Recent Trends in Europe's Output and Productivity Growth Performance at the Sector Level, 2002-2015. International Productivity Monitor, (33), 8-23.

Wiener, N. (1956). The theory of prediction. Modern mathematics for engineers, 1 , 125-139. 